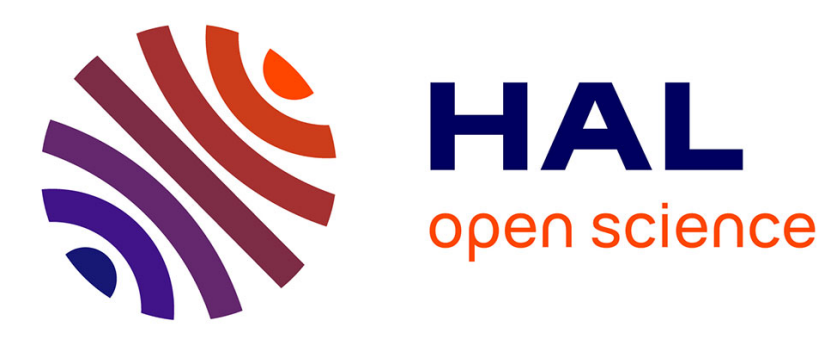

\title{
Analyse diachronique de la croissance du réseau ferroviaire français entre 1860 et 1930
}

Christophe Mimeur, Thomas Thévenin

\section{To cite this version:}

Christophe Mimeur, Thomas Thévenin. Analyse diachronique de la croissance du réseau ferroviaire français entre 1860 et 1930 : Entre expansion connexionniste et sélection hiérarchique?. Flux - Cahiers scientifiques internationaux Réseaux et territoires, 2020, 122 (4), pp.69-87. 10.3917/flux1.122.0069 . hal-03128119

\section{HAL Id: hal-03128119 \\ https://hal.science/hal-03128119}

Submitted on 26 Jul 2021

HAL is a multi-disciplinary open access archive for the deposit and dissemination of scientific research documents, whether they are published or not. The documents may come from teaching and research institutions in France or abroad, or from public or private research centers.
L'archive ouverte pluridisciplinaire HAL, est destinée au dépôt et à la diffusion de documents scientifiques de niveau recherche, publiés ou non, émanant des établissements d'enseignement et de recherche français ou étrangers, des laboratoires publics ou privés. 


\title{
Analyse diachronique de la croissance du réseau ferroviaire français entre 1860 et 1930 : entre expansion connexionniste et sélection hiérarchique?
}

\author{
Christophe Mimeur \\ Thomas Thévenin
}

\section{INTRODUCTION}

Entre 1830 et 1930 le paysage des villes et campagnes françaises a été façonné par la construction du réseau ferroviaire. L'année 1930 marque l'apogée du chemin de fer français pour atteindre 50000 kilomètres de voies. Cette date constitue également une rupture historique avec les premières faillites d'entreprises, la chute des compagnies de chemin de fer d'intérêt local, jusqu'à la création de la SNCF en 1937. Ainsi en moins d'un siècle, le réseau s'est effacé pour atteindre aujourd'hui moins de 28000 kilomètres de voies. Nombreuses sont les cartes qui illustrent la rapidité avec laquelle s'est opéré le phénomène d'extension, puis de rétraction de cette lourde infrastructure visible dans les paysages français. Elles montrent surtout l'importance de l'évolution structurelle sur le fonctionnement du réseau jusqu'à aujourd'hui. La théorisation des réseaux (Dupuy, Offner, 2005) suggère pourtant que la dynamique et la logique du réseau compte autant que sa structure. À cet égard, Studeny (1995) rappelle que, très tôt, « les vitesses élevées ne valent que pour les grandes liaisons, dès que l'on quitte les lignes principales, un net ralentissement s'opère ». Le principe d'universalité, au fondement de la conception du réseau ferroviaire, n'est donc pas aussi clair, ce qui suggère à certains auteurs que la croissance d'un réseau de transport se manifeste par une organisation qui lui est spécifique (Xie, Levinson, 2007).

Pour éclairer ces différents points de vue, nous postulons que l'accélération de la vitesse implique une hiérarchie du réseau qui crée des disparités temporelles (durée des trajets) et surtout, augmente les inégalités spatiales (proximité à une gare). Dans cette perspective, cet article propose de revisiter l'expansion du réseau ferroviaire français dans une perspective géohistorique et quantitative. À cette fin, la base de données FRANcE (French RAilway NEtwork), qui recense les dates de création des lignes sous la forme d'un système d'information géographique, sera mobilisée. Cette source sera tout d'abord utilisée pour identifier les "lois quantitatives d'évolution du réseau » (Dupuy, 1991). Dans un second temps, nous reviendrons sur la constitution du graphe ferroviaire entre 1860 et 1930, ainsi que sur la grille de lecture adoptée dans l'optique d'une caractérisation de la hiérarchie du réseau. Dans un dernier temps, il s'agit d'étudier en quoi la croissance du réseau ferroviaire répond à des principes universels identifiés par la science des réseaux et comment la hiérarchisation du réseau ferroviaire français se manifeste dans les territoires traversés.

\section{LA CROISSANCE DES RÉSEAUX EN QUESTION}

La formalisation d'une théorie territoriale des réseaux est apparue dans les années 1980 (Dupuy, 1987). Pour le chercheur et l'aménageur, elle paraît d'autant plus nécessaire que l'infrastructure de transport s'inscrit dans une temporalité longue, qui va jusqu'à une " permanence » (Dupuy, 1991) et laisse des traces durables dans les territoires, justifiant la focale du temps long. Pourtant, les premières analyses diachroniques se sont heurtées à la difficulté de réunir des données suffisamment exhaustives et ce sont des extrapolations qui ont permis dans un 
premier temps de formaliser un modèle de croissance macroscopique de réseau, vers une diffusion limitée au départ puis une accélération de la diffusion du réseau (Dupuy, 1991). Cette théorie s'appuie néanmoins sur trois entrées : une structure, une dynamique, une logique, qu'il s'agit de mettre à l'épreuve de la réalité des faits ou d'essais empiriques.

\section{Une structure souvent abordée par des considérations topologiques}

La structure d'un réseau est le résultat d'une «morphologie d'agencement des lieux » (Dupuy, Offner, 2005). Son analyse suppose le changement d'une métrique topographique vers une métrique topologique (Lévy, Lussault, 2003). Les lieux deviennent des nœuds et les lignes sont celles qui permettent de les lier. Le simple formalisme des graphes permet de bien rendre compte de cette propriété des réseaux à relier des ressources et les tentatives de modélisation n'ont pas manqué (Haggett, Frey, Cliff, 1977). Les célèbres indicateurs de Kansky (1963) ont été mobilisés par de nombreuses disciplines pour relater la taille et la complexité d'un réseau.

Plus récemment, Ducruet et Beauguitte (2014) ont listé un nombre important de travaux dont certains décrivent l'évolution d'un réseau de transport (Bretagnolle, 2009; Dancoisne, 1984). Ces travaux de référence introduisent des indicateurs plus géographiques, tels que l'éloignement moyen du graphe et la distance moyenne entre tous les sommets du graphe, alors même que les premières études de réseau entreprises par les physiciens ou les sociologues ignoraient toute notion de distance. La question de l'évolution du réseau à proprement parler et des principes qui régissent son extension connaît moins de succès (Xie, Levinson, 2008).

\section{Une dynamique qui confronte nécessairement le réseau et le territoire}

Si l'analyse macroscopique des points et des lignes suggère bien une évolution qui suit une courbe logistique (Garrison, Levinson, 2014), l'étude de la dynamique du réseau suggère qu'il «serait sujet à des cycles de développement qui feraient apparaître des temps forts pour des espaces forts » (Dupuy, Offner, 2005), venant accréditer l'idée que «les réseaux de transport [...] entretiennent [...] les anisotropies du territoire ». Ces « lignes de force » chères à Lévy et Lussault (2003) alimentent régulièrement le débat sur les effets structurants des infrastructures de transport (Offner, 1993, 2014). L'étude de l'extension des réseaux, en lien avec les espaces qu'ils desservent, fait l'objet d'une riche littérature souvent proche de la science régionale ou de l'économie historique. Atack et alii (2010) identifient des effets macroéconomiques entre la croissance d'un réseau de transport et le développement régional à partir de simples dotations en infrastructures. Plus récemment, Hornung (2015) a tenté d'identifier des effets microéconomiques de l'arrivée d'un nouveau réseau dans la croissance démographique des villes, grâce à un recours croissant aux systèmes d'information géographique (Kasraian et alii, 2016). Ces outils permettent de confronter des variables linéaires ou ponctuelles du réseau à des variables linéaires ou zonales des territoires, grâce à des modélisations économétriques. L'accès au réseau, variable binaire, est souvent la seule qui permet d'estimer l'effet de la diffusion de réseaux de transport, qu'ils soient autoroutiers aux États-Unis (BaumSnow, 2007 ; Duranton, Turner, 2012) ou ferroviaires (Gregory, Henneberg, 2010; Schwartz, Gregory, Marti-Henneberg, 2011), ou encore sur des terrains asiatiques (Chi, 2010 ; Ji et alii, 2014).

Pour autant, cette dynamique reste souvent topologique et s'appuie sur l'hypothèse d'une homogénéité des infrastructures. Jannelle (1969) propose un nouveau regard sur cette dynamique du réseau à travers une "demande d'accessibilité ». Les innovations du transport permettent alors de tendre vers une " convergence spatio-temporelle»: cette quête d'accessibilité repose sur un «nouveau rapport fondé sur la circulation, le flux, la vitesse » (Dupuy, 1991). Nous identifions deux pistes encore peu explorées : peu d'essais historiques quantifiés existent pour démontrer l'évolution hiérarchisée d'un réseau de transport, en raison essentiellement des difficultés à mobiliser des données suffisamment riches et précises (Delaplace, 2014). Par ailleurs, la « vitesse différenciée » (Ollivro, 2000) a encore peu été intégrée dans l'analyse de la croissance d'un réseau de transport et dans son rapport avec le territoire (Bretagnolle, 2009 ; Kasraian et alii, 2016). Elle constitue pourtant une nouvelle métrique topologique des relations entre les lieux et bouleverse les configurations existantes: " C'est le temps de parcours qui compte et non la distance» (Riboud, 1981). Or, l'idée d'une diffusion du réseau, véhiculée par l'extension topologique du réseau, est ici mise en défaut par «l'évidence trompeuse d'une vitesse généralisée » (Flonneau, Guigueno, 2009). L'innovation ferroviaire, comme d'autres, doit être mise au regard d'une dynamique faite de ruptures et de permanences qui rend chaque réseau plus ou moins adaptatif sur le temps long. 


\section{Une logique propre au réseau ferroviaire ?}

Dupuy et Offner (2005) font l'hypothèse de « lois d'évolution, [de] logiques semblables pour différents types de réseaux en fonction de leur forme, de leur structure, des systèmes techniques utilisés ». À propos de la croissance du réseau ferroviaire français, il semblerait que des considérations techniques et financières aient été des facteurs parmi d'autres, à l'origine de l'établissement de « lignes » de la part des compagnies privées françaises au XIXe siècle (Ribeill, 1986). Les lignes les plus rentables constituent alors un "premier réseau ». Les autres bénéficient d'un montage financier différent et sont reléguées comme lignes secondaires, tandis qu'un "troisième réseau » fait l'objet d'autres garanties financières spécifiques. Il semble ainsi que les grandes compagnies privées conçoivent le plan de leurs « lignes », sans que l'établissement global d'un réseau soit pensé (Lepetit, 1986).

Ici, l'étude sur la longue durée de la croissance du réseau ferroviaire français participe à la « vérification de certaines lois d'organisation des réseaux » (Ducruet, 2010), dont les effets du temps long se font ressentir durablement. Elle vise à qualifier la hiérarchie dans le réseau dans sa dimension topologique (par l'extension physique du réseau) et sa dimension cinétique (par l'investissement différencié dans l'infrastructure qui se traduit par des différentiels de vitesse). Le recours aux indicateurs locaux (Encart 1) permet d'étudier dans quelle mesure des caractéristiques locales du réseau ont un impact sur la structure et la dynamique globale du même réseau, en faisant émerger des "éléments de compréhension nouveaux concernant le fonctionnement, les forces et les faiblesses des réseaux étudiés » (Gleyze, 2005).

En associant la structure, la cinétique et l'adaptabilité du réseau (Dupuy, Offner, 2005), ce travail permet un regard renouvelé sur la hiérarchisation du réseau ferroviaire français à plusieurs titres. Le premier apport est la prise en compte d'une hétérogénéité de l'infrastructure à partir de la modélisation de la vitesse sur l'ensemble du territoire et pendant toute la période de croissance du réseau. Par ailleurs, l'étude diachronique des réseaux présente souvent des difficultés d'évaluation et de représentation (Dupuy, 1991) : le recours aux SIG et au formalisme des graphes permet la construction d'indicateurs rendant compte des dimensions topologiques et cinétiques de la croissance du réseau ferroviaire français.

\section{Encart 1. Qualifier la structure et les performances d'un réseau}

Connexité : elle vérifie si une suite d'arcs permet de relier l'ensemble des nœuds. Jusque dans les années 1850, le réseau ferroviaire français n'est pas connexe. Par extension, elle peut définir aussi l'extension du réseau.

Centralité de degré : il s'agit de la mesure la plus classique de l'importance d'un nœud dans le réseau. Elle permet de compter le nombre de branches divergentes partant d'un nœud (Bretagnolle, 2003 ; Pumain, 1982). 1 signifie que la gare est un terminus de ligne, 2 signifie que la gare voit le simple passage d'une ligne, 3 et plus signifie que la gare se situe dans une situation de carrefour, plus ou moins complexe selon le nombre de branches. Cette variable illustre aussi la capacité d'une gare à avoir un accès direct à un nombre plus ou moins important d'autres destinations par la seule infrastructure.

Connectivité : elle permet de repérer les éléments « incontournables du réseau » (Ducruet, Beauguitte, 2014) ainsi que l'existence d'itinéraires alternatifs en croisant les informations topologiques et cinétiques

Intermédiarité : c'est un indicateur de connectivité qui permet de caractériser l'importance d'un nœud ou d'un arc dans le réseau au regard de tous les autres : cet indicateur permet de répondre aux problématiques d'adaptation de l'offre et de management des risques (To, 2016). Sur le temps long, la centralité d'intermédiarité permet de montrer des logiques de renforcement, de bouleversement et de sélection de tout ou partie d'un réseau au profit ou au détriment d'un autre. Elle mesure le nombre de plus courts chemins qui passent par un nœud ou par un arc.

Circuité : issue de la théorie du circuit, elle permet de repérer et d'identifier s'il existe des solutions alternatives performantes au regard de l'ensemble des itinéraires possibles entre deux sommets d'un réseau. 


\section{LA CROISSANCE DU RÉSEAU FERROVIAIRE PAR LE PRISME DE LA VITESSE}

La plupart des systèmes d'information géohistoriques collectent des informations ponctuelles, dont le caractère rare et hétérogène rend leur manipulation complexe (Gregory, Ell, 2007). Les Britanniques ont été précurseurs dans la constitution $d^{\prime}$ un système d'information géohistorique à partir de l'Atlas du Colonel Cobb, pour numériser le linéaire du réseau ferroviaire britannique à l'occasion d'une initiative européenne (MorillasTorné, 2012). L'utilisation des SIG peut permettre la construction de nouveaux indicateurs dans la caractérisation de réseaux historiques, mais cette démarche reste encore discrète dans le champ des études de long terme (Kasraian et alii, 2016).

\section{La base de données FRANcE}

La base de données FRANcE est un instrument de recherche qui permet de lier des informations sur un système de transport et sur les caractéristiques du territoire qu'il dessert. Elle est constituée d'un SIG qui retrace l'ensemble du linéaire du réseau ferroviaire français depuis le début du XIXe siècle. À la différence du cas britannique qui s'appuyait sur une seule source d'information, la reconstitution du réseau français s'appuie sur différentes sources permettant d'obtenir des informations démographiques à l'échelle de la commune ainsi que des vitesses moyennes associées à chaque tronçon du réseau (Mimeur, 2016 ; Mimeur et alii, 2017 ; Thévenin et alii, 2016).

Nous avons fait le choix de ne retenir le réseau qu'entre 1860 et 1930 (Figure 1) : avant cette date, le réseau n'est pas connexe et les mesures sur le graphe perdent alors de leur intérêt. En l'espace de soixante-dix ans, le réseau est ainsi multiplié par cinq. En début de période, l'ensemble des lignes relevant de la loi Legrand, promulguée en 1842, sont construites. Dans le texte de loi, elles sont décrites comme des lignes qui permettent de relier les principaux foyers démographiques et économiques du pays (Ribeill, 1985). S'ensuit alors l'ouverture de nombreuses lignes secondaires jusqu'en 1878, date de l'adoption du plan Freycinet, qui place 181 lignes dans la loi du 17 juillet 1879, et qui prévoit la desserte par le rail de chaque sous-préfecture et chef-lieu de canton. L'étude de ce seul réseau de transport dans des analyses de moyenne et longue distances se justifie par le caractère " hégémonique » du chemin de fer, que Ribeill (1985) hiérarchise en "grands axes, axes secondaires et axes-affluents locaux ». Durant cette période, les progrès techniques ont permis également d'augmenter la vitesse moyenne des trains sur certains tronçons, en fonction des investissements inégalement consentis par les six grandes compagnies de l'époque mais aussi en fonction des besoins pour le transport des marchandises, dont le poids est conséquent dans l'écosystème ferroviaire du XIXe siècle. Ce travail ne s'appuie en revanche que sur l'infrastructure. II ne prend en compte ni la diversité des services, tels que les trains express ou les trains omnibus, ni la fréquence de passage des trains, faute d'informations exhaustives et suffisamment précises pour la période d'étude. Ainsi, sur la base de l'évolution de l'infrastructure, nous proposons de construire des indicateurs permettant de rendre compte de cette hiérarchisation, dans les dimensions structurelle, dynamique et logique du réseau ferroviaire.

\section{Le formalisme des graphes pour mesurer la hiérarchie du réseau}

L'apport de la base de données FRANcE réside dans la transformation du SIG-H (Système d'Information Géo-Historique) en un graphe fonctionnel, qui permet de modéliser les gares comme des nœuds et les tronçons comme des arcs. De cette sorte, il est facile d'étudier l'évolution de la topologie du réseau sur le temps long, à travers la connexité (Encart 1), en reprenant des indicateurs (Tableau 1, colonne a) qui sont classiquement utilisés dans l'évolution de la dotation en infrastructures (Kasraian et alii, 2016). Sur la longue durée, on peut confronter certaines caractéristiques des nœuds en fonction de l'ancienneté de leur connexion au réseau pour identifier des phénomènes d'appropriation du réseau. Nous utiliserons également un indicateur de centralité (Encart 1) : la centralité de degré est la plus classique.

Ces seuls indicateurs supposent une infrastructure homogène, le long de laquelle on se déplace à une vitesse homogène, et ne permet de traduire ni l'accélération ni les différentiels de vitesse sur l'ensemble du réseau. La base de données FRANcE permet de tenir compte de la vitesse en prenant en compte une infrastructure hétérogène qui se lit dans la différenciation des valeurs des arcs, non pas à partir de distances euclidiennes classiques mais de temps de parcours nécessaire pour traverser l'arc. Cela permet de calculer des itinéraires et de déterminer des indicateurs d'accessibilité grâce au classique algorithme de plus court chemin (Mimeur et alii, 2017). Il permet aussi de s'intéresser à la configuration des réseaux (Lhomme, 2015) en utilisant la centralité d'intermédiarité (Encart 1). Dans ce travail, elle est calculée en fonction de la seule distance euclidienne supposant une homogénéité de l'infrastructure, et en 
Figure 1. La croissance du réseau ferroviaire français entre 1860 et 1930

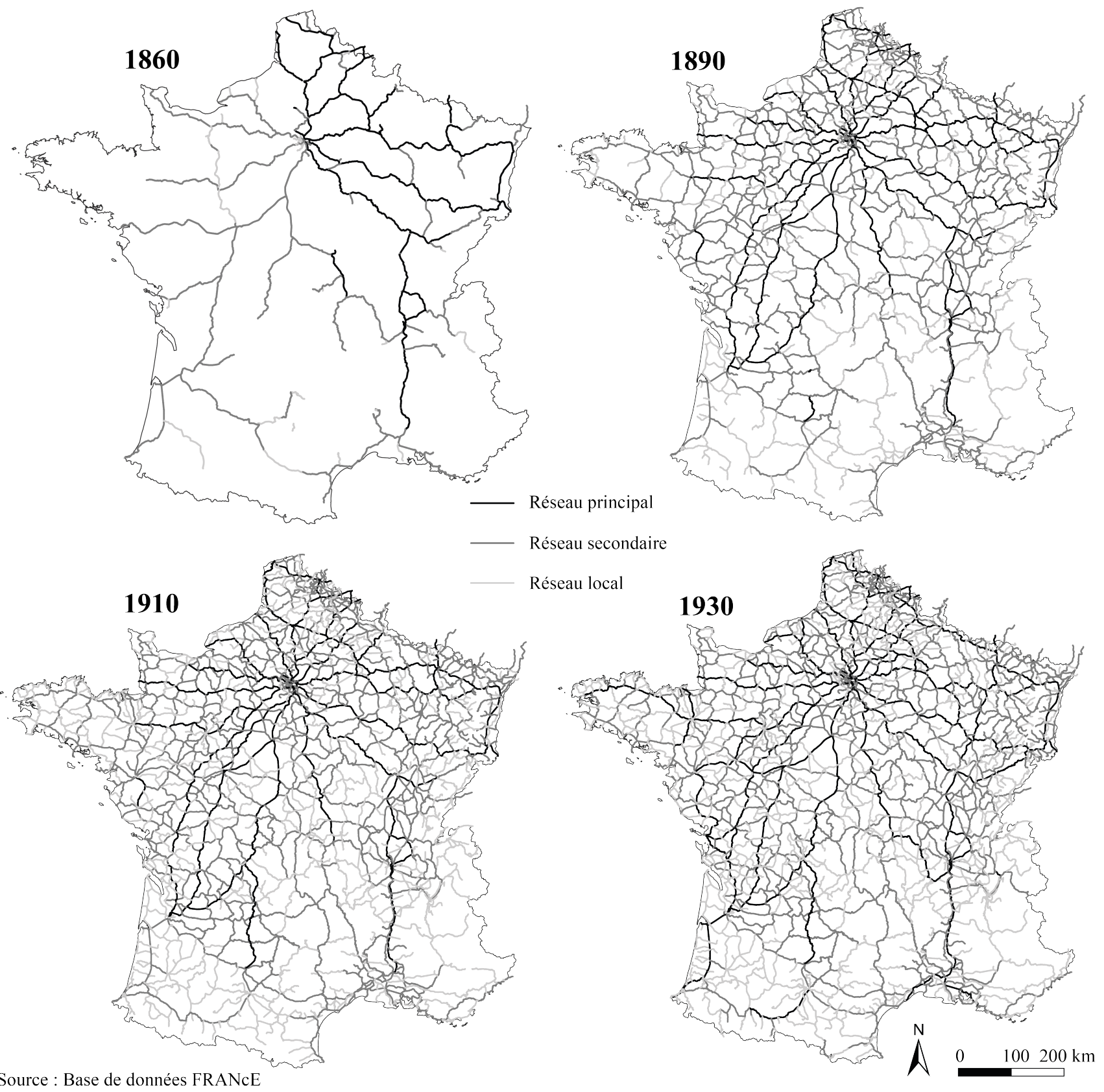

Source : Base de données FRANcE

fonction de la vitesse permise par l'infrastructure. La mesure des écarts (Tableau 1, colonne b) permet de voir les lieux de passage des plus courts chemins qui profitent de la vitesse différenciée (Ollivro, 2000) et au contraire ceux qui voient une convergence de passage des plus courts chemins moindre que ce qu'une infrastructure homogène supposerait.

Enfin, pour appréhender la dimension logique du réseau ferroviaire français sur le temps long, nous nous appuyons 
Tableau 1. Les indicateurs du graphe pour analyser la hiérarchie du réseau par ses points et ses lignes

\begin{tabular}{|c|c|c|c|}
\hline & a. Structure & b. Dynamique & c. Logique \\
\hline $\begin{array}{l}\text { Caractéristique } \\
\text { du réseau associé }\end{array}$ & $\begin{array}{c}\text { Connexité et ancienneté } \\
\text { de la connexion }\end{array}$ & $\begin{array}{c}\text { Vitesse différenciée et } \\
\text { hiérarchisation du réseau }\end{array}$ & $\begin{array}{c}\text { Connectivité et circulation } \\
\text { dans le réseau }\end{array}$ \\
\hline \multirow{3}{*}{ Indicateur } & $\begin{array}{l}\text { Connexion au réseau } \\
\begin{array}{c}1 \text { = gare } \\
0=\text { sans gare }\end{array}\end{array}$ & $\begin{array}{l}\text { Centre d'intermédiarité par la } \\
\text { distance euclidienne pour l'arc i } \\
\qquad C_{B e u c}(i)=\frac{g_{\text {euc } j k}(i)}{g_{\text {euc } j k}} \\
\text { où } g_{\text {euc } j_{j k}}(i) \text { est le nombre de plus } \\
\text { courts chemins passant par l'arc } \\
\text { i et } g_{\text {euc } j k} \text { le nombre total de plus } \\
\text { courts chemins }\end{array}$ & $\begin{array}{l}\text { Distance par le plus court chemin } \\
\qquad \text { Dist }_{s p}(i)=\frac{\sum_{j}^{N} D \min _{i j}}{N_{j}} \\
\text { où } \sum_{j}^{N} D \min _{i j} \text { est la somme des } \\
\text { plus courts chemins entre i et chaque } \\
\text { nœud j et } N_{i j} \text { le nombre de nœuds j }\end{array}$ \\
\hline & $\begin{array}{l}\text { Centralité de degré } \\
\text { pour le nœud } \mathbf{i} \\
C_{D}(i)=\sum_{j}^{N} x_{i j} \\
\text { où } \mathrm{N} \text { est le nombre de } \\
\text { nœuds, } \mathrm{j} \text { l'ensemble des } \\
\text { autres nœuds et } x_{i j} \text { une } \\
\text { relation adjacente entre i et } \mathrm{j}\end{array}$ & $\begin{array}{l}\text { Centralité d'intermédiarité par } \\
\text { la vitesse moyenne pour l'arc i } \\
\qquad C_{B v i t}(i)=\frac{g_{v i t}(i)}{g_{v i t} j_{j k}} \\
\text { où } g_{v i t_{j k}}(i) \text { est le nombre de plus } \\
\text { courts chemins passant par l'arc } \\
\text { i et } g_{v i t_{j k}} \text { le nombre total de plus } \\
\text { courts chemins }\end{array}$ & $\begin{array}{c}\text { Distance de résistance } \\
\text { Dist }_{r e s}(i)=\sum_{j}^{N} D_{i j} \\
\text { où } \sum_{\text {proportionnellement avec la }}^{N} D_{i j} \text { augmente } \\
\text { distance entre i et j et diminue } \\
\text { proportionnellement avec le nombre } \\
\text { de chemins différents entre i et j }\end{array}$ \\
\hline & & & 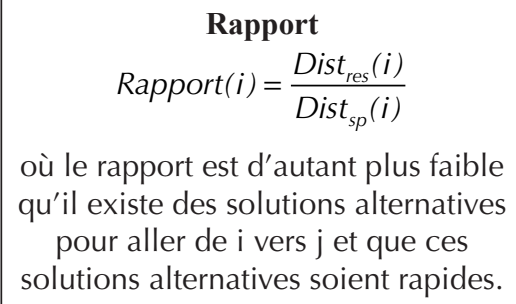 \\
\hline
\end{tabular}

sur des indicateurs de connectivité. Ils caractérisent un nœud par rapport à l'ensemble des autres nœuds du réseau.. Parallèlement, nous construisons un indicateur de résistance pour chaque nœud, issu de la matrice des distances de résistance, qui prend en compte tous les chemins alternatifs au plus court chemin. Issu de la théorie du circuit (Encart 1), il traduit la redondance de chemins entre deux points. Dès lors, une structure de réseau en arbre suppose des valeurs proches entre la distance par le plus court chemin et la distance de résistance, étant donné le caractère non redondant d'une telle structure. À l'inverse, nous construisons un indicateur qui évalue le rapport entre ces deux mesures (Tableau 1, colonne c). Si cet indicateur tend vers 0 , alors il traduit l'ajout de voies parallèles, donc une circuité dans le réseau. Cela vient donc renforcer la connectivité des lieux bénéficiant $d^{\prime}$ une telle évolution. Le regard porté sur cet indicateur permet ainsi d'analyser des logiques de cohérence à l'intérieur du réseau, alors que d'autres sont potentiellement écartées de ce phénomène.

La dernière partie de ce travail permet la mise en œuvre de ces mesures pour qualifier la hiérarchie du réseau ferroviaire français dans le temps et dans l'espace. 


\section{UNE HIÉRARCHISATION NODALE ET RÉTICULAIRE}

La hiérarchie est définie en géographie comme une " forme d'organisation d'un système en sous-systèmes tels que le nombre de sous-systèmes varie selon une progression géométrique inverse du nombre des éléments de chaque sous-système » (Hypergéo). La forme de la hiérarchie urbaine correspond à cette définition (Pumain, 1997). Pour chacun des critères de structure, dynamique et logique du réseau, nous interrogeons le cas du réseau ferroviaire français pour étudier la forme de hiérarchie suite à son extension entre 1860 et 1930.

\section{Une coévolution entre connexité et population}

Alors que l'endogénéité des relations entre transport et territoire est le ciment des difficultés à faire émerger des phénomènes de causalité, plusieurs études ont conclu à la coévolution des deux systèmes, à partir de données simples et souvent partielles (Bretagnolle, 2003 ; Pumain, 1982). La coévolution suggère que l'extension du réseau et la croissance urbaine s'entretiennent l'une et l'autre et renforcent les positions relatives des villes.
Par la lunette de la topologie du réseau, nous cherchons ici à identifier cette coévolution entre la croissance du réseau ferroviaire d'une part et l'évolution de la population d'autre part. II s'agit de voir aussi comment le réseau se hiérarchise et dans quelle mesure cette hiérarchie se rapproche de la hiérarchie urbaine bien connue (Pumain, 1997). Nous observons les niveaux de population des communes, relativement à l'ancienneté de leur connexion au réseau (Tableau 2), en posant I'hypothèse que les communes desservies le plus tôt sont les plus peuplées et que la diffusion du réseau se fait de manière inversement proportionnelle au niveau de peuplement des communes desservies.

Cette hypothèse se confirme dans la mesure où, en moyenne, une commune desservie en 1870 compte 2379 habitants contre 732 habitants en 1920. En valeur moyenne, on voit que pour une décennie donnée, les écarts sont beaucoup plus importants pour les villes les plus anciennement connectées : 4351 habitants pour celles connectées depuis dix à vingt ans en 1880 et 6548 habitants pour celles connectées depuis soixante à soixante-dix ans en 1930. Ces écarts ont tendance à chuter dès que I'on considère des communes connectées

Tableau 2. Population moyenne des communes desservies entre 1860 et 1930, selon l'ancienneté de la connexion en décennie

\begin{tabular}{|c|c|c|c|c|c|c|c|c|c|}
\hline & & \multicolumn{8}{|c|}{ Graphe ferroviaire } \\
\hline & & 1860 & 1870 & 1880 & 1890 & 1900 & 1910 & 1920 & 1930 \\
\hline \multirow{8}{*}{$\begin{array}{c}\text { Ancienneté } \\
\text { de la connexion } \\
\text { au réseau }\end{array}$} & 1 an & 5634 & 2379 & 1593 & 1332 & 1066 & 1118 & 732 & \\
\hline & 10 ans & & 6029 & 2564 & 1630 & 1354 & 1055 & 1031 & 721 \\
\hline & 20 ans & & & 6915 & 2656 & 1684 & 1355 & 993 & 1007 \\
\hline & 30 ans & & & & 7520 & 2807 & 1695 & 1321 & 995 \\
\hline & 40 ans & & & & & 8123 & 2875 & 1685 & 1343 \\
\hline & 50 ans & & & & & & 8513 & 2978 & 1728 \\
\hline & 60 ans & & & & & & & 9063 & 3185 \\
\hline & 70 ans & & & & & & & & 9733 \\
\hline
\end{tabular}

Source : Base de données FRANcE 
dix ans plus tard. Ces chiffres nous servent à montrer deux éléments. Le réseau a d'abord desservi des communes dont le niveau de population était déjà élevé. Les valeurs sont très dispersées à l'intérieur de chaque groupe de villes, ce qui s'explique par une grande diversité des situations et démontre la précaution avec laquelle la notion d'effet structurant doit être abordée. Par ailleurs, le différentiel de population moyenne entre ces communes desservies depuis longtemps et le reste des autres communes desservies s'est accentué sur le temps long entre 1860 et 1930. On constate donc que le réseau s'est diffusé progressivement vers des communes dont la taille était de plus en plus petite.

D'autre part, une lecture diagonale permet de suivre l'évolution des niveaux de population d'un même échantillon de villes. On constate que le niveau moyen de population des communes connectées avant 1890 a tendance à augmenter sur I'ensemble de la période. En revanche, celles qui sont connectées ensuite connaissent une évolution contrastée, voire une diminution : ce sont des communes qui ont profité du plan Freycinet qui a consisté en la desserte de communes très peu peuplées, et dont la dynamique démographique semble plus chaotique (1). À ce stade, l'étude de la connexité par la succession des graphes entre 1860 et 1930 permet de constater que l'extension du réseau ferroviaire tend à reproduire la hiérarchie urbaine et que ce phénomène se renforce avec le temps, en dépit d'une forte disparité des situations attestées par des écartstypes très importants.

Si d'un point de vue temporel, la coévolution entre extension du réseau et hiérarchie urbaine semble assez évidente (Bretagnolle, 2003), les indicateurs de centralité du graphe permettent de qualifier autrement la connexité au réseau. La Figure 2 analyse la distribution de la population selon la centralité de degré. La population a été transformée en logarithme et discrétisée par la méthode des écarts-types. Pour une année donnée, la population est représentée par cinq « boîtes à moustaches", des communes les moins peuplées aux communes les plus peuplées. On représente le minimum, le premier quartile, la moyenne, le troisième quartile et le maximum, les valeurs extrêmes étant représentées par les points. Nous ne nous intéressons ici qu'aux communes de plus de 2500 habitants en fin de période, dans la mesure où la dispersion des valeurs ne rend pas interprétable les résultats pour les communes de moins de 2500 habitants. Entre 1860 et 1880, on remarque que les communes les plus peuplées sont surtout de simples étapes (2 branches divergentes) et des carrefours à 3 branches, qui représentent tout de même plus 75\% des valeurs en 1860 . Le réseau n'est pas encore suffisamment complexe pour identifier des valeurs plus hautes, même si des valeurs extrêmes au-dessus de 5 apparaissent dès 1870. À partir de 1880, on observe une distinction plus claire entre les villes qui possèdent en moyenne un carrefour à quatre branches et celles qui ne sont que des étapes: les premières correspondent aux plus peuplées quand les secondes sont les moins peuplées. Entre elles, certaines villes plus moyennes deviennent des carrefours importants, dans une classe intermédiaire où les cas sont plus contrastés. On constate donc ici une relation parallèle entre la complexité du carrefour et le niveau de population des communes de 2500 habitants. Là aussi, les écarts entre les communes les plus peuplées et les moins peuplées tendent à s'agrandir au fil du temps, accréditant l'hypothèse d'une hiérarchisation progressive du réseau.

\section{Un renforcement des radiales}

Si la partie précédente montre que le réseau s'est diffusé en suivant la hiérarchie urbaine, nous cherchons ici à qualifier la hiérarchie propre à l'infrastructure, à travers la dynamique du réseau imposée par les vitesses différenciées. Nous choisissons ici de confronter deux visions hiérarchiques du réseau. La première s'appuie sur la topologie du réseau et l'agencement du réseau en fonction des distances euclidiennes, la seconde s'appuie sur le même agencement du réseau mais en appliquant des vitesses différenciées. Nous cherchons à identifier les tronçons du réseau sur lesquels les itinéraires optimaux passent le plus souvent selon cette double entrée. Dans un premier temps, nous étudions la distribution du nombre d'itinéraires optimaux passant par chaque arête du graphe. Entre 1860 et 1930, elle correspond à une distribution à progression géométrique, telle que nous l'avons déjà définie.

Dans le tableau 3, nous cherchons à estimer un périmètre de forme d'une distribution de Pareto de l'intermédiarité dans le réseau, selon la topologie basée sur la distance euclidienne d'une part, et celle basée sur la vitesse moyenne d'autre part. Plus ce paramètre est important, plus la hiérarchie est forte et les écarts entre le haut et le bas de la distribution sont forts. Dans les deux cas, on constate que la croissance du réseau ferroviaire s'accompagne d'une accentuation de la hiérarchie entre 1860 et 1930. Cela traduit la « capacité de cette structure à s'organiser et à fonctionner en réseau »(Bavoux et alii, 2005), 
Figure 2. La centralité de degré dans le graphe en fonction de la population, classée selon une discrétisation logarithmique par écart-type

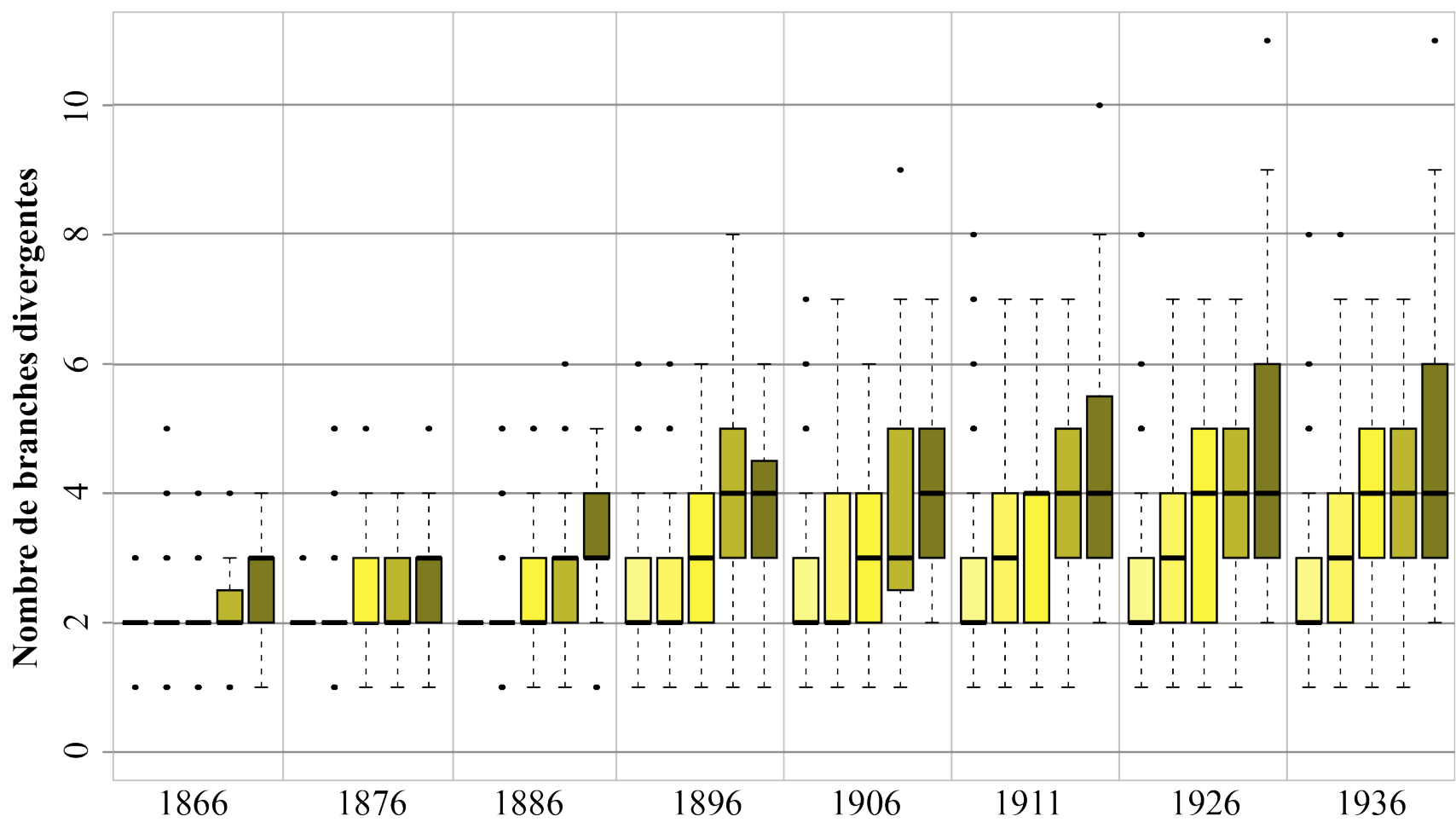

Discrétisation de la population logarithmique par écart type

Source : Base de données FRANcE

qu'en 1860 : la valeur du paramètre de la forme de la distribution de Pareto s'est accentuée de 2,62\% entre 1860 et 1870. Cette hiérarchisation augmente à partir de 1890, alors qu'un vaste réseau d'intérêt local et peu rapide émerge. On remarque d'ailleurs qu'entre 1870 et 1920, la hiérarchisation imposée par la vitesse se renforce beaucoup plus vite que la hiérarchisation imposée par la seule topologie du réseau, en témoigne l'écart grandissant de la dernière colonne du tableau 3. Ainsi, la confrontation entre la distance topologique et la vitesse suggère que la différenciation de la vitesse accentue bien la hiérarchisation du réseau et qu'elle se renforce jusqu'en 1920.

S'appuyer sur des indicateurs locaux du graphe permet de rendre compte de cette hiérarchisation dans l'espace, ainsi que de la sélection opérée, qui renforce la position de certains tronçons. Partant de I'hypothèse qu' « aucune ligne n'offrait un avantage matériel décisif»(Bavoux, 1994), nous pouvons vitesse font finalement que le réseau est davantage hiérarchisé 
Tableau 3. Estimation du paramètre de forme d'une distribution de Pareto de l'intermédiarité dans le réseau entre 1860 et 1930, selon une topologie basée sur la distance euclidienne et une topologie basée sur la vitesse moyenne

\begin{tabular}{|c|c|c|c|c|}
\hline & & $\begin{array}{l}\text { Paramètre de forme de la } \\
\text { distribution de Pareto }\end{array}$ & $\begin{array}{c}\text { Évolution par décennie } \\
(\%)\end{array}$ & $\begin{array}{c}\text { Écart de " hiérarchisation " } \\
\text { entre vitesse et situation } \\
\text { contrefactuelle }\end{array}$ \\
\hline \multirow{8}{*}{$\begin{array}{l}\text { Topologie basée } \\
\text { sur la distance } \\
\text { euclidienne }\end{array}$} & 1860 & 0,56 & - & \\
\hline & 1870 & 0,47 & $-16,10$ & \\
\hline & 1880 & 0,54 & 15,88 & \\
\hline & 1890 & 0,70 & 28,49 & \\
\hline & 1900 & 0,75 & 7,86 & \\
\hline & 1910 & 0,77 & 2,87 & \\
\hline & 1920 & 0,84 & 8,60 & \\
\hline & 1930 & 0,87 & 3,53 & \\
\hline \multirow{8}{*}{$\begin{array}{l}\text { Topologie basée } \\
\text { sur la vitesse moyenne }\end{array}$} & 1860 & 0,52 & - & $-0,04$ \\
\hline & 1870 & 0,53 & 2,62 & 0,06 \\
\hline & 1880 & 0,66 & 24,24 & 0,12 \\
\hline & 1890 & 0,88 & 34,08 & 0,19 \\
\hline & 1900 & 1,05 & 18,80 & 0,30 \\
\hline & 1910 & 1,11 & 5,82 & 0,34 \\
\hline & 1920 & 1,22 & 10,30 & 0,38 \\
\hline & 1930 & 1,20 & $-2,43$ & 0,32 \\
\hline
\end{tabular}

interpréter ces écarts comme des choix des compagnies de l'époque. En superposant les deux valeurs sur la Figure 3, on peut identifier les tronçons qui se trouvent favorisés par la vitesse différenciée (noir plus épais sur la Figure 3a) et ceux qui se retrouvent en retrait de par les choix opérés (gris plus épais sur la Figure 3b). 1930 correspond à l'apogée du réseau ferroviaire français, qui compte plus de $50000 \mathrm{~km}$. En dépit d'un maillage important, les itinéraires optimaux à partir de la vitesse (Figure 3b) imposent une structure en arbre, caractéristique de la plupart des réseaux ferrés (Bavoux et alii, 2005). Les axes sur lesquels la vitesse a un effet positif sont ceux qui partent de Paris, vers Le Mans, vers Tours, vers Lille, vers I'Est ainsi que des liaisons transversales qui rejoignent I'Atlantique à la Méditerranée. Ce sont donc surtout des radiales autour de Paris, mais également d'autres axes qui partent de carrefours importants, tels ceux de Lyon, Saint-Étienne, Strasbourg, Dijon, Mulhouse, Rennes, Toulouse. Par ailleurs, cette figure rend compte aussi d'arbitrages : à l'Est, l'axe Paris-Belfort est investi 
Figure 3. Centralité d'intermédiarité des arcs en 1930 (a. selon la distance euclidienne au premier plan, b. selon la vitesse au premier plan)

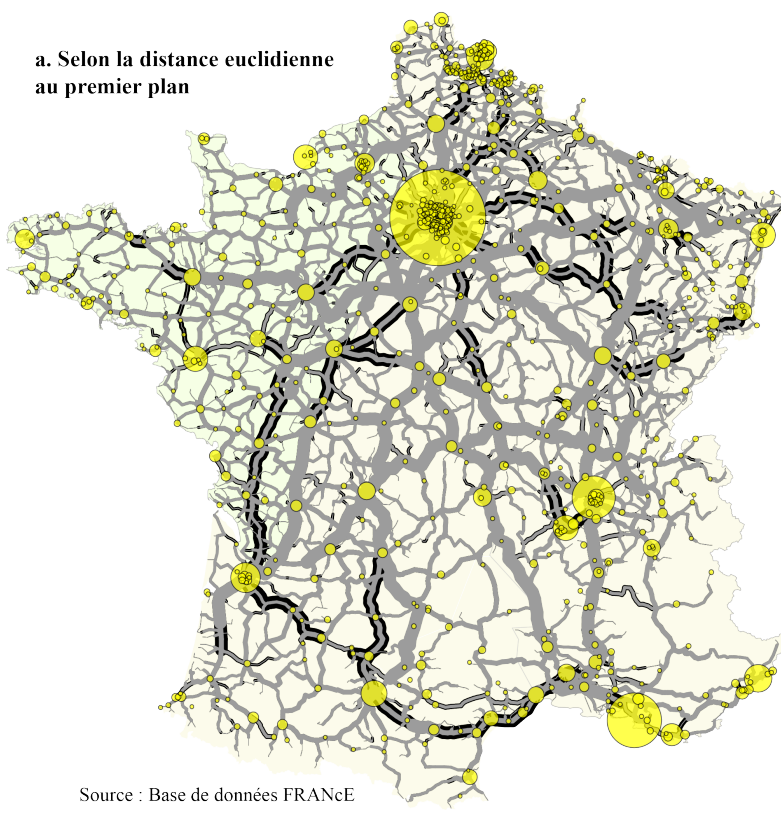

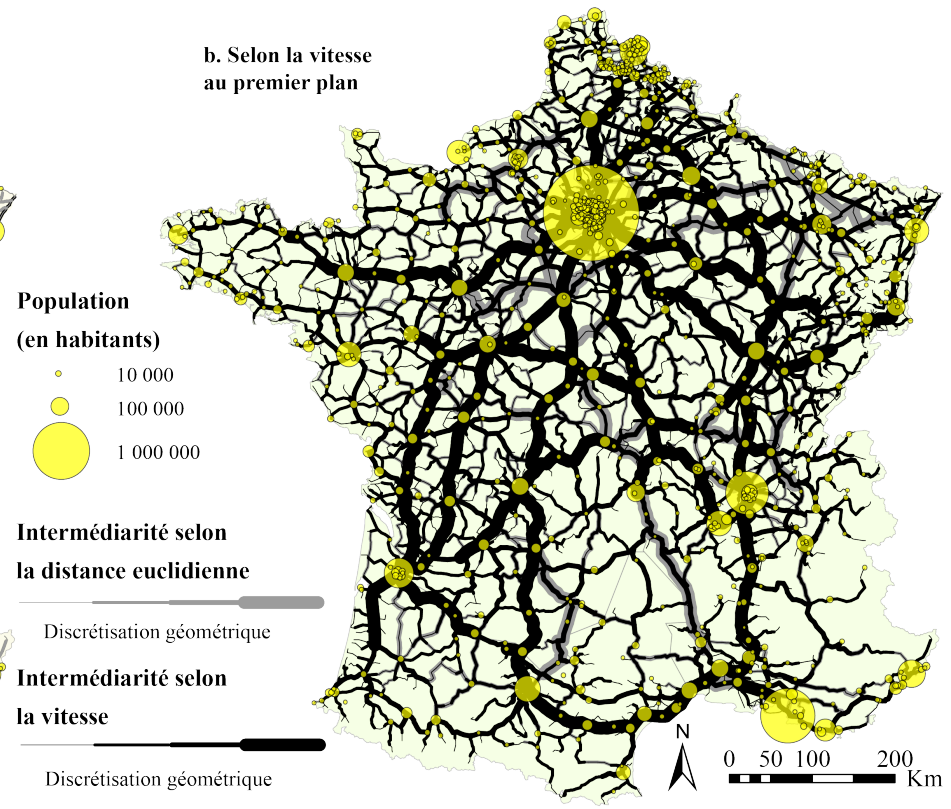

tandis que le modèle contrefactuel suggère un axe optimal qui rejoint Nancy et Metz depuis le Sud-Est de la région parisienne. Par un investissement sur l'axe Paris-Nevers, la compagnie du PLM se trouve en meilleure position par rapport à la compagnie du Paris-Orléans pour rejoindre Clermont-Ferrand, quand les itinéraires optimaux contrefactuels favorisent un passage par Orléans et Bourges. À l'Ouest, deux axes à vitesse élevée suggèrent de rejoindre Bordeaux par Niort ou par Angoulême.

À l'inverse, la Figure 3b montre que les choix se sont faits parfois au détriment de tronçons qui ont vu leur nombre d'itinéraires optimaux diminuer par rapport à la situation contrefactuelle. Il s'agit le plus souvent d'axes transversaux. La plupart d'entre eux se situent dans une ceinture assez éloignée de Paris : on lit bien ici les efforts consentis sur les axes radiaux alors que les axes transversaux servaient davantage à lier des lignes entre elles plutôt qu'à y attacher un service performant. Par ailleurs, des axes parallèles aux principales radiales sont "pénalisés » par une vitesse qui tombe lourdement. Enfin, la traversée du Massif Central ne semble pas faire l'objet de choix positifs en termes de vitesse : les itinéraires optimaux suggèrent alors de contourner ce massif par la Vallée du Rhône ou par I'axe Limoges-Toulouse. Celui-ci ressort de manière évidente avec la différenciation par la vitesse.
Au final, si les gares se diffusent de manière parallèle à l'évolution de la hiérarchie urbaine, l'infrastructure se diffuse selon un processus tout aussi hiérarchique. Une hiérarchie s'impose, privilégiant les axes radiaux au détriment d'axes transversaux, tout en favorisant aussi d'autres axes issus de décisions stratégiques des compagnies. Parmi elles, on peut faire l'hypothèse que les grandes compagnies raisonnaient également à des échelles plus locales, où le maillage vient non seulement améliorer la connexité, mais aussi la connectivité de certains nœuds du réseau par rapport à tous les autres. On peut aussi postuler que les compagnies puissent avoir été elles-mêmes influencées par des considérations de politiques locales. Une confrontation avec des sources en provenance des compagnies et de la presse régionale peut être une piste de prolongation de ces hypothèses.

\section{Une circulation régionalisée ?}

En dépit d'une hiérarchisation du réseau par la connexité et la connectivité, plus ou moins exacerbée par des vitesses différentes, le réseau ferroviaire français est le plus étendu en Europe au début du XXe siècle (Martí-Henneberg, 2013). Son maillage se renforce pendant toute sa phase de croissance et fait apparaître potentiellement des chemins alternatifs entre 
deux points. Cette sous-partie se propose d'étudier, dans le temps et dans l'espace, quels territoires profitent le plus de ce maillage. Le Tableau 4 illustre ce phénomène. Pour chaque gare du réseau, l'indicateur DSP/DRES représente le rapport entre le plus court chemin entre deux points et l'ensemble des chemins possibles entre ces deux mêmes points. Ce rapport est en moyenne de 0,59 en 1860 et tombe à 0,18 en 1930 : pendant ces soixante-dix ans, le nombre de chemins entre deux points a augmenté, et certains de ces chemins sont bien efficaces. Le Tableau 4 met en regard ce rapport moyen avec l'ancienneté de la connexion de la gare au réseau. Deux éléments sont à relever. Le premier est que plus une gare est ancienne, plus elle bénéficie du maillage du réseau : le rapport est toujours plus bas. Le second élément est l'augmentation des écarts entre les gares nouvellement desservies et les gares les plus anciennes : encore une fois, la hiérarchisation progressive du réseau fait son œuvre et malgré la densification du réseau au cours du temps, les chemins alternatifs efficaces bénéficient davantage aux gares les plus anciennes, laissant entrevoir une prime au haut de la hiérarchie urbaine. Pour autant, voit-on l'émergence d'ensembles régionalisés dans lesquels on peut faire l'hypothèse $d^{\prime}$ 'une circulation locale, qui bénéficierait à davantage de population?
La Figure 4 illustre la répartition du rapport entre le plus court chemin entre deux points et l'ensemble des chemins possibles entre ces deux mêmes points vers 1930, au regard de la population des communes desservies directement par une gare. Plus la valeur de ce rapport est faible, plus la gare bénéficie de possibilités de circuler efficacement avec des alternatives. Inversement, une valeur élevée signifie une dépendance à un chemin, caractéristique des réseaux en arête de poisson. On constate une égale répartition de ce rapport (2). Nous nous intéressons dans un premier temps aux valeurs plus faibles, qui traduisent une possibilité de circuler localement. Elles se concentrent de deux manières différentes: elles forment des graphes de taille petite ou moyenne à l'Est de la France et un ensemble plus homogène dans le quart nord-ouest.

Les valeurs faibles forment parfois des grappes de petite taille : on les retrouve assez naturellement en région parisienne avec un réseau maillé. Une large région lilloise ressort également, qui démontre l'existence d'un tissu industriel fin, en dépit d'une population parfois en bas de la hiérarchie urbaine. D'autres grappes d'ampleur plus modeste apparaissent enfin : autour de Strasbourg, de Nancy, de Lyon. Cet indicateur permet de montrer que, à côté des grandes relations interurbaines plus ou moins favorisées par la vitesse, la configuration du réseau

Tableau 4. Évolution du rapport DSP/DRES en fonction de l'ancienneté de la connexion en décennie

\begin{tabular}{|c|c|c|c|c|c|c|c|c|c|}
\hline & & \multicolumn{8}{|c|}{ Rapport DSP/DRES } \\
\hline & & 1860 & 1870 & 1880 & 1890 & 1900 & 1910 & 1920 & 1930 \\
\hline \multirow{8}{*}{$\begin{array}{l}\text { Ancienneté de la } \\
\text { connexion au réseau }\end{array}$} & 1 an & 0,5998 & 0,4065 & 0,2922 & 0,2419 & 0,2247 & 0,2185 & 0,2031 & \\
\hline & 10 ans & & 0,3856 & 0,2942 & 0,2308 & 0,2167 & 0,2078 & 0,2048 & 0,2051 \\
\hline & 20 ans & & & 0,2811 & 0,2319 & 0,2078 & 0,2002 & 0,1938 & 0,2065 \\
\hline & 30 ans & & & & 0,2226 & 0,2092 & 0,1937 & 0,1895 & 0,143 \\
\hline & 40 ans & & & & & 0,2007 & 0,1926 & 0,1835 & 0,19 \\
\hline & 50 ans & & & & & & 0,1874 & 0,1828 & 0,1829 \\
\hline & 60 ans & & & & & & & 0,1785 & 0,1827 \\
\hline & 70 ans & & & & & & & & 0,1782 \\
\hline
\end{tabular}


Figure 4. Rapport DRES/DSP dans le réseau de 1930,

l'intermédiarité des arcs selon la vitesse en 1930 est proposée en arrière plan

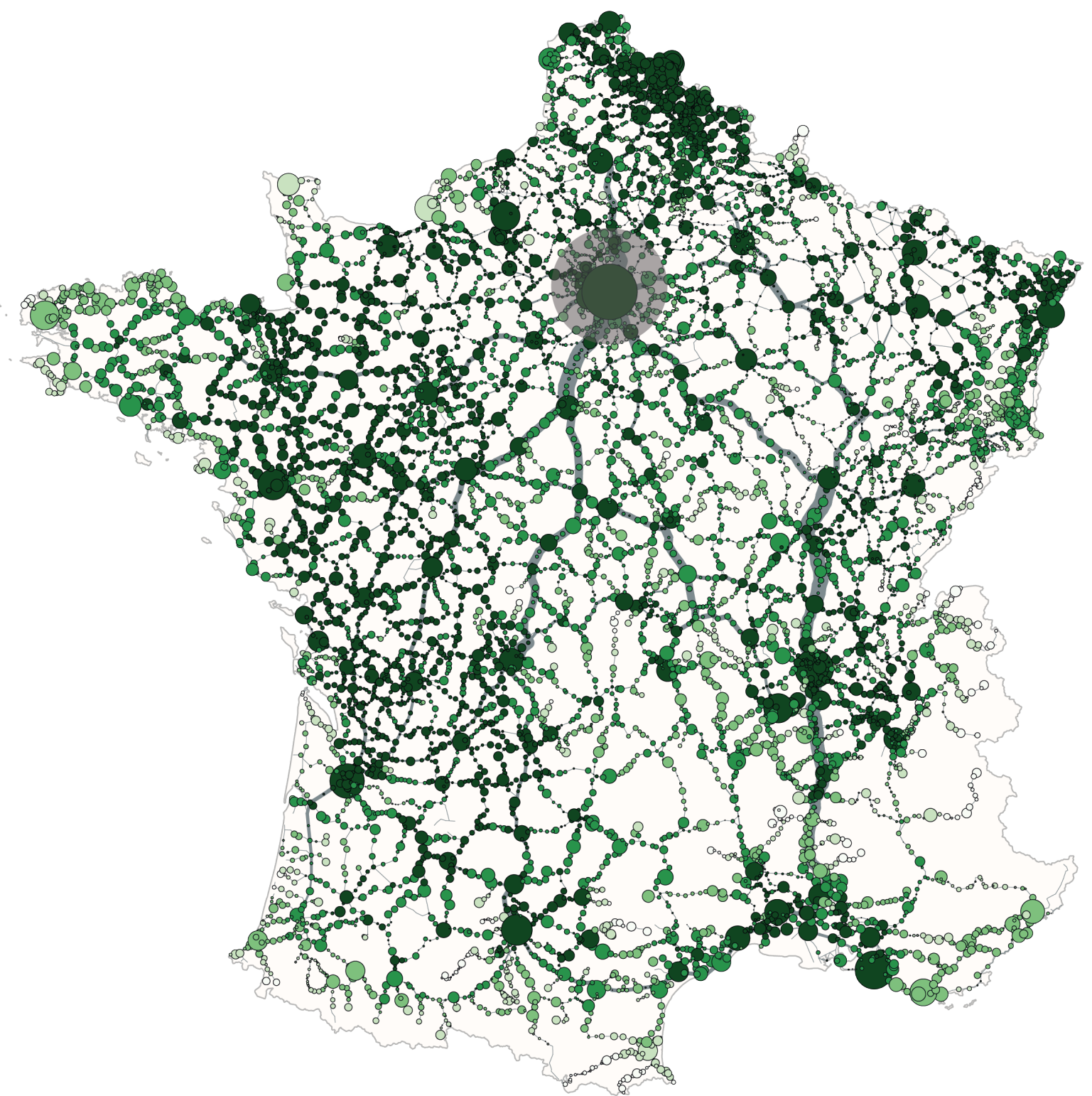

\section{Population}

(en habitants)

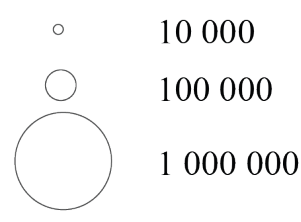

Rapport DRES / DSP

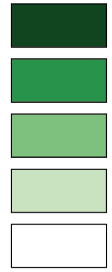

$0,15-0,17$

$0,18-0,19$

$0,20-0,22$

$0,23-0,26$

$0,27-0,36$

\section{Intermédiarité selon}

la vitesse

Discrétisation géométrique

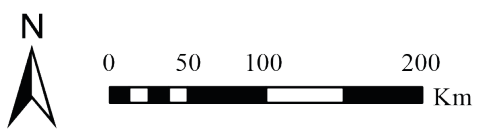

Source : Base de données FRANcE 
Figure 5. Évolution du rapport DRES/DSP entre 1890 et 1930, l'intermédiarité des arcs selon la vitesse en 1930 est proposée en arrière-plan

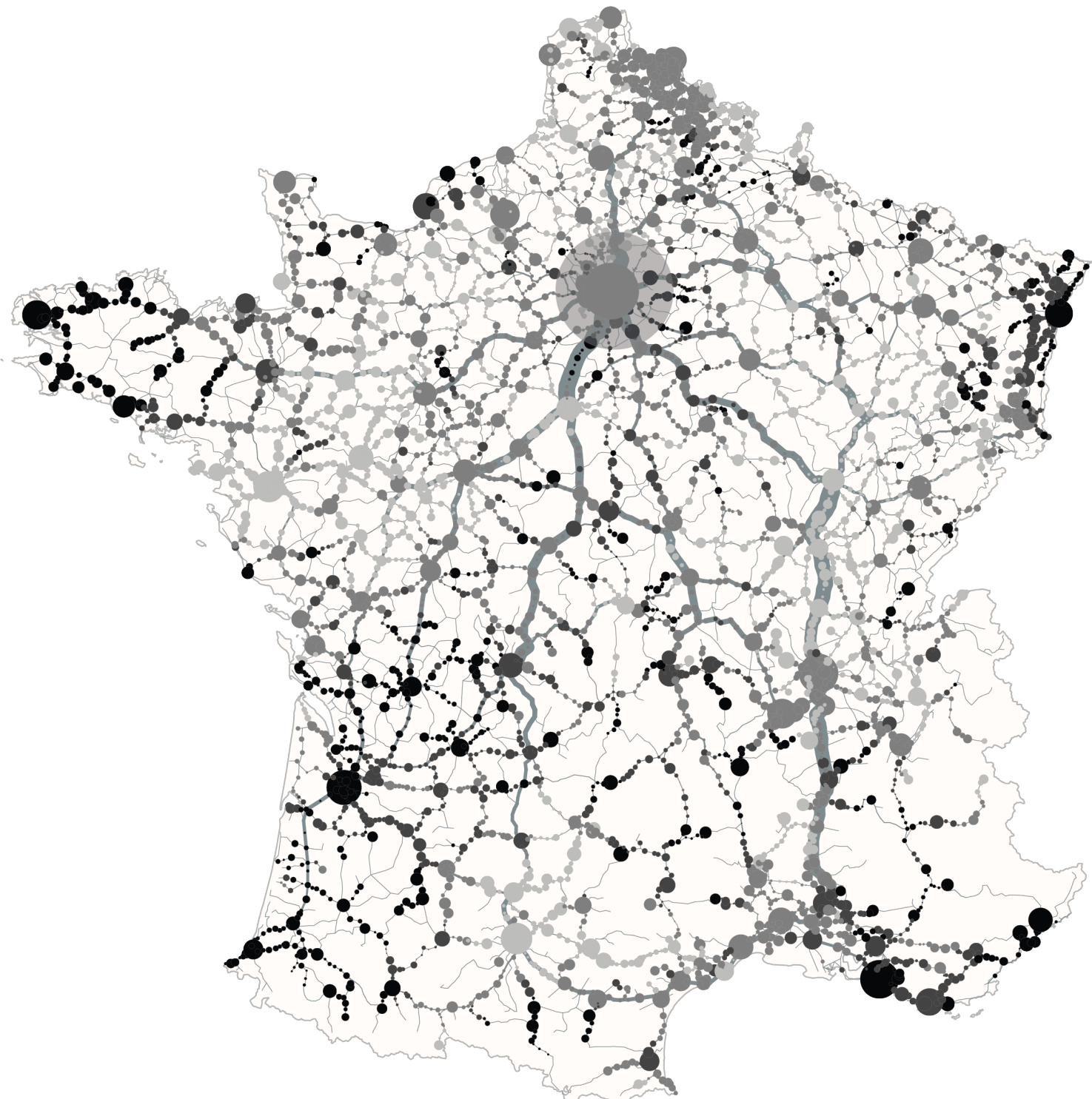

Population

(en habitants)

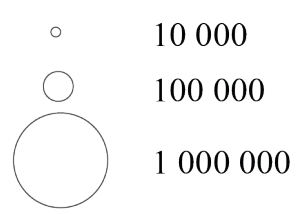

Evolution DRES / DSP (\%)

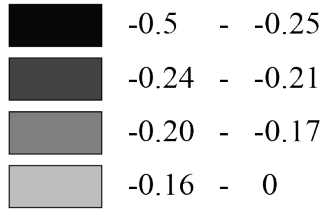

\section{Intermédiarité selon}

la vitesse

Discrétisation géométrique

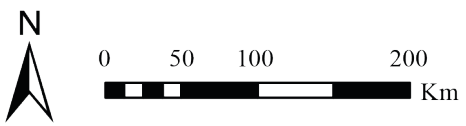

Source : Base de données FRANcE 
de ces zones permet des chemins alternatifs efficaces, qui ne sont pas entièrement dépendants des principaux axes radiaux qui les desservent par ailleurs. Dès lors, on constate que la forte hiérarchisation du réseau à l'échelle nationale parvient à cohabiter avec des configurations locales favorables à une circulation efficace. Ces zones dessinent toutefois des ensembles très peuplés, où le haut de la hiérarchie urbaine reste à chaque fois l'épicentre de cette configuration locale, mais dont les effets de la connectivité du réseau ne se limitent pas à la seule proximité des grandes villes.

Ces valeurs faibles ont aussi une large tendance à se concentrer dans l'ouest de la France: les valeurs sont tirées vers l'extrême de la distribution autour de Rennes, de Nantes, d'Angers, du Mans, de Tours, de Poitiers et de Bordeaux, relevant des mêmes considérations exposées juste au-dessus. Pour autant ces valeurs ont tendance à se propager au-delà de la périphérie proche de ces villes, ce que l'on analyse par la coexistence d'axes secondaires, dont la hiérarchie est moins écrasante que dans l'Est de la France (Figure 3b).

La Figure 5 propose d'analyser l'évolution du rapport DRES/DSP entre 1890 et 1930 . Plus la valeur du rapport chute dans le temps, plus la gare a vu se multiplier les possibilités de circuler, au regard de la population des communes directement desservies. Seules les gares qui préexistaient en 1890 sont donc représentées ici : toutes ont connu une amélioration depuis 1890. Entre les deux dates, c'est surtout le réseau local qui s'est renforcé, tandis que d'autres axes ont vu leur vitesse augmenter.

Les espaces qui ont le plus profité de cette période sont inégalement répartis. En Alsace encore, de nouvelles ramifications secondaires ont fait chuter le rapport, renforçant la possibilité de chemins alternatifs. C'est bien la possibilité d'effectuer des boucles qui renforce la connectivité d'un lieu : le désenclavement de Digne-les-Bains vers Cannes en est l'illustration, qui apporte de nouvelles solutions pour circuler dans la région. On observe la même chose en Bretagne, où seule la desserte littorale existait en 1890. Par ailleurs, on voit que la densification du réseau dans le bassin aquitain, encore rare en 1890, a considérablement amélioré les possibilités de circuler, et encore davantage autour d'Angoulême, Périgueux et Bordeaux. En région parisienne, l'émergence de lignes transversales au sud et à l'est de Paris améliore la circuité dans ces zones. Pour résumer, la principale amélioration se produit au sein des ensembles les plus larges que l'on a évoqués avec la situation de 1930.
En comparaison, les grappes les plus restreintes autour des grandes villes sont celles qui ont connu l'évolution la moins forte : la circulation y était donc déjà performante en 1890. Cette figure permet donc de différencier au moins deux rythmes dans la logique du réseau à l'échelle locale : le maillage du réseau peut traduire une cohérence d'ensembles territorialisés, surtout autour des grandes villes, dans un périmètre somme toute assez réduit et très tôt dans la phase de croissance du réseau. Cela a aussi été le cas pour l'ensemble plus large du bassin industriel du Nord et du triangle Rennes-NantesTours. Jusqu'en 1930, la densification du réseau permet quant à elle un rattrapage dans certains espaces, dans la mesure où l'évolution du rapport permet d'identifier des ensembles cohérents cette fois-ci à une échelle plus large, régionale comme en Alsace ou dans le Bassin Aquitain.

En 1930, le réseau a atteint son apogée. Malgré un maillage maximum, la circulation est surtout privilégiée dans un certain nombre de "sous-réseaux », dont la répartition paraît hiérarchisée dans le temps et dans l'espace : autour des grandes villes très tôt, puis dans des espaces régionaux entre 1890 et 1930.

\section{Discussion}

De nombreuses contributions portant sur l'évolution des réseaux tentent $d^{\prime}$ identifier dans leurs analyses les marques d'un système de transport innovant, d'un développement territorial et des choix de politiques publiques (Cats et alii, 2020). Des éléments de contextualisation participent à l'identification de ces marqueurs (Pumain, 2014). Notre contribution vise précisément à utiliser la base de données FRANcE et le formalisme des graphes pour identifier ces marqueurs à l'aune de la croissance du réseau ferroviaire français.

Notre travail nous a permis de mesurer l'importance de ces trois dimensions dans la croissance du réseau ferroviaire français, se lisant à nos yeux à différents niveaux de hiérarchisation du réseau. Au plus tôt, la dimension topologique semble décisive dans la création d'un réseau connexe qui favorise le haut de la hiérarchie urbaine. L'accès précurseur au réseau concerne les places urbaines les plus dynamiques en termes démographiques jusqu'en 1930, par-delà les modifications incrémentales ultérieures du réseau, attestant non seulement de la congruence des croissances du réseau et de la démographie mais aussi de celle de leur inertie sur le temps long. Notre analyse vient confirmer que la structure du réseau s'accommode de situations démographiques préexistantes (Pumain, 1982). 
La dimension cinétique, à travers la vitesse différenciée, propose une vision exhaustive du territoire sur l'ensemble de la période. En dépit d'un maillage qui gagne I'ensemble de la France, le «net ralentissement» (Studeny, 1995) en-dehors des axes principaux entérine une hiérarchisation des axes, qui s'accentue au cours du temps. Ces choix stratégiques révèlent I'intérêt des grandes compagnies de l'époque de développer un réseau ferré hiérarchisé et centralisé sur la capitale. Pour autant, ces mêmes choix peuvent parfois conduire à des liaisons rapides parfois inattendues, de par leur positionnement dans le réseau. De ce point de vue, nous posons l'hypothèse que la circulation de trains de marchandises peut participer à d'autres choix de configuration des réseaux. Enfin, la dernière dimension permet d'analyser l'évolution de la connectivité en traversant les échelles. Par la recherche d'itinéraires alternatifs, nous avons pu voir émerger des sous-ensembles pouvant s'apparenter à des sous-réseaux, dont les échelles ne sont pas les mêmes pendant toute la période. On les identifie dès la fin du XIX ${ }^{\mathrm{e}}$ siècle autour des principales villes, telles que Lyon, SaintÉtienne, Strasbourg, Dijon ou Rennes, tandis que la dernière phase de croissance du réseau voit l'apparition d'ensembles régionalisés. Ce phénomène ne concerne pas l'ensemble du territoire et subit des rythmes différents : la différenciation de I'infrastructure par la vitesse s'ajoute de manière décisive à la structure du réseau.

La base de données FRANcE permet alors de dresser une " fresque » du développement du chemin de fer entre 1860 et 1930, en identifiant des places fortes ainsi que des lignes fortes, aux échelles nationales et régionales. Sans doute, les perspectives de ce travail nous incitent à poursuivre l'exploitation de cette base dans I'identification de nouvelles hypothèses mettant en scène des phénomènes beaucoup plus locaux. Cellesci, dont certaines ont déjà été évoquées au cours du texte, suggèrent non seulement une diversification méthodologique mais aussi un recours à l'interdisciplinarité. En effet, le développement du réseau ferroviaire a fortement marqué I'histoire économique, industrielle et sociale du pays : tant l'historien que l'économiste ont tout à fait leur place dans ce processus de construction de connaissances.

Par extension, I'utilisation des méthodes mixtes ainsi que l'apport des autres sciences humaines et sociales doivent pouvoir permettre d'améliorer la source. L'une des originalités de cette base est la prise en compte d'une infrastructure hétérogène en fonction de la vitesse, quand de nombreuses analyses font I'hypothèse d'une infrastructure homogène. En revanche, l'une des principales limites est qu'elle ne tient pas compte du niveau de service offert sur l'infrastructure. Si les relations express permettent de rejoindre des points importants du réseau rapidement, les relations omnibus présentent des conditions plus dégradées qui sont certainement un autre marqueur de la forte hiérarchie dans le réseau ferroviaire français, qui peut prendre alors des allures de sélection des territoires (Bretagnolle, 2003). Ces différentiels de service se retrouvent également dans la notion de fréquence de passage : I'attractivité d'une ligne n'est pas qu'une fonction de la vitesse à laquelle on circule dessus. Pour cela, le recours à d'autres archives, dont l'extraction d'informations suggère un travail conséquent, est envisageable, tout au moins à des échelles locales dans un premier temps.

L'extension de ce travail suggère également $d^{\prime}$ interroger le rôle stratégique des acteurs du ferroviaire dans le façonnement d'un réseau aussi hiérarchisé. Entre les stratégies des grandes compagnies, le clientélisme politique et les dimensions techniques inhérentes à l'infrastructure, le croisement entre cette base de données et de nouvelles sources doit permettre d'apporter de nouveaux éléments à l'appréhension du processus de mise en réseau et de l'extension de noyaux de développement économique.

Enfin, la croissance du réseau ferroviaire s'inscrit dans une dynamique globale de progrès technique et d'extension des réseaux routiers et fluviaux qui concerne toute l'Europe. Même si le chemin de fer a une forme d'hégémonie dans la seconde moitié du XIX ${ }^{\mathrm{e}}$ siècle, le développement parallèle de ces réseaux suggère des phénomènes de concurrence et de complémentarité entre les modes de transport. Cela ouvre de nouvelles perspectives au-delà de 1930, à I'heure où la démocratisation de la route participe au démantèlement du réseau ferroviaire : la grille de lecture de cette contribution peut également permettre d'alimenter un travail sur l'étude de la suppression de "barreaux » entiers du réseau et leurs conséquences sur la consistance du réseau dans son ensemble. Des études comparées en Europe, en fonction des données disponibles (Martí-Henneberg, 2017), sont une perspective de recherche dans la construction de nouvelles hypothèses. Ce travail s'inscrit alors dans une démarche géohistorique émergente en France, qui vise à se doter d'un instrument de recherche permettant d'explorer les mutations socio-économiques à différentes échelles. 
Christophe Mimeur est Maître de conférences en Géographie à CY Cergy Paris-Université au sein du laboratoire MRTE. Ses travaux portent sur l'analyse des interactions entre réseau et territoire à partir de la caractérisation des réseaux de transport. Il étudie les infrastructures dans la longue durée mais aussi les dynamiques territoriales liées à la reconfiguration des services de transport. christophe.mimeur@cyu.fr
Thomas Thévenin est professeur de géographie à I'Université de Bourgogne Franche-Comté, chercheur à I'UMR ThéMA et directeur adjoint de la MSH de Dijon. Ses travaux portent sur la représentation et l'analyse des données spatiale et temporelle. Ces objets d'étude sont ainsi focalisés sur les infrastructures dans le temps long et la mobilité quotidienne. thomas.thevenin@u-bourgogne.fr

\section{Notes}

(1) Nous avons calculé les mêmes indicateurs en ne prenant seulement en compte que les communes de plus de 2500 habitants : les tendances sont similaires, hormis la lecture diagonale à partir de 1890, qui, à l'inverse du cas général, ne montre pas de différences avec les diagonales débutant avant 1890. Enfin, la seule prise en compte des communes de moins de 2500 habitants ne montre pas de hiérarchie claire. Nous posons l'hypothèse que les effets de l'Exode Rural et d'évolution globale de la démographie participent à l'absence de hiérarchie dans les communes de moins de 2500 habitants.

(2) L'indicateur Dist(sp), le dénominateur du rapport, présente une distribution dissymétrique attestant une forte hiérarchisation. Cet indicateur est sensible aux effets de bord, comme on pouvait s'y attendre: les distances au plus court chemin sont plus longues à mesure que l'on s'écarte du centre géométrique de l'espace traité. Sur le temps long, sa décroissance est linéaire. L'indicateur Dist(res), le numérateur du rapport propose lui aussi une distribution dissymétrique mais l'effet de bord n'est pas marqué : il est plus sensible à son environnement proche. Sa décroissance n'est pas linéaire et suit davantage une courbe logarithmique, que I'on retrouve dans les autres indicateurs du graphe, tels ceux d'intermédiarité.

\section{BibLIOGRAPHIE}

Atack J., Bateman F., Haines M., Margo R. A., 2010, Did Railroads Induce or Follow Economic Growth? Urbanization and Population Growth in the American Midwest, 1850-1860, Social Science History, Vol. 34, $\mathrm{n}^{\circ} 2$, p. 171-197. DOI: $10.2307 / 40587344$

BAum-SnOw N., 2007, Did highways cause suburbanization?, The Quarterly Journal of Economics, p. 775-805.

Bavoux J.-J., 1994, Le "carrefour" bourguignon : analyse d'un espace de circulation, Paris : CNRS éditions.

Bavoux J.-J., Beaucire F., Chapelon L., Zembri P., 2005, Géographie des transports, Paris : A. Colin.

Bretagnolle A., 2003, Vitesse des transports et sélection hiérarchique entre les villes françaises, in: Pumain D., Mattei M.-F. (dir), Données urbaines, 4, Paris: Anthropos, p. 309-323.

Bretagnolle A., 2009, Villes et réseaux de transport : des interactions dans la longue durée (France, Europe, Etats-Unis), HDR de géographie, Paris : Université Panthéon-SorbonneParis I.

Cats O., Vermeulen A., Warnier M., Van Lint H., 2020, Modelling growth principles of metropolitan public transport networks, Journal of Transport Geography, Vol. 82, p. 102567. DOI: 10.1016/j.jtrangeo.2019.102567

CHI G., 2010, The Impacts of Highway Expansion on Population Change: An Integrated Spatial Approach, Rural Sociology, Vol. 75, n 1, p. 58-89. DOI: 10.1111/j.15490831.2009.00003.x
Dancolsne P., 1984, Théorie des graphes et constitution du réseau ferré francais, Thèse de géographie, Paris: Paris I-Sorbonne.

DelaplaCE M., 2014, Dessertes ferroviaires à grande vitesse : des effets structurants toujours... la pensée et l'action publique, L'Espace Géographique, Vol. 43, n²1, p. 51-67.

DuCRuet C., 2010, Les mesures locales d'un réseau. Document de travail [en ligne] disponible à l'adresse : https://halshs.archives-ouvertes.fr/halshs-00546814v2 (consulté le 23 novembre 2020)

Ducruet C., Beauguitte L., 2014, Spatial Science and Network Science: Review and Outcomes of a Complex Relationship, Networks and Spatial Economics, Vol. 14, n 3, p. 297-316. DOI: 10.1007/s11067-013-9222-6

Dupuy G., 1987, Vers une théorie territoriale des réseaux: une application au transport urbain, Annales de Géographie, Vol. 96, p. 658-679.

Dupur G., 1991, L'urbanisme des réseaux: théories et méthodes, Paris : Armand Colin.

Dupuy G., Offner J.-M., 2005, Réseau: bilans et perspectives, Flux, 2005/4 ( $\left.n^{\circ} 62\right)$, p. 38-46.

Duranton G., Turner M. A., 2012, Urban Growth and Transportation, Review of Economic Studies, Vol. 79, n 4 , p. 1407-1440. DOI: 10.1093/restud/rds010

Flonneau M., Guigueno V. (dir.), 2009, De l'histoire des transports à l'histoire de la mobilité, Rennes: Presses universitaires de Rennes. 
Garrison W. L., LeVInSON D. M., 2014, The Transportation Experience: Policy, Planning, and Deployment, New York: Oxford University Press.

GleYze J.-F., 2005, La vulnérabilité structurelle des réseaux de transport dans un contexte de risques, Thèse de géographie, Université Paris-Diderot - Paris VII. [En ligne] Disponible à I'adresse: https://tel.archives-ouvertes.fr/tel-00138991 (consulté le 13 avril 2020).

Gregory I. N., ElL P. S., 2007, Historical GIS: technologies, methodologies, and scholarship, [s.l.]: Cambridge University Press, UK. [En ligne] Disponible à l'adresse : http://books. google.fr/books?hl=fr\&lr=\&id=I_HhhJF5Un4C\&oi=fnd\&pg $=$ PA $1 \& d q=$ gregory+ell\&ots $=7 p$ XIm2Ezcy $\&$ sig $=$ NuRavNNh P9PriYawqX5d0-WQx-U > (consulté le 30 avril 2013).

Gregory I. N., Henneberg J. M., 2010, The Railways, Urbanization, and Local Demography in England and Wales, 1825-1911, Social Science History, Vol. 34, $\mathrm{n}^{\circ} 2$, p. 199-228.

Haggett P., Frey A. E., Cliff A. D., 1977, Locational Analysis in Human Geography, New York: John Wiley \& Sons.

Hornung E., 2025, Railroads and Growth in Prussia, Journal of the European Economic Association, Vol. 13, n 4, p. 699736. DOI: 10.1111/jeea.12123

JANELLE D. G., 1969, Spatial reorganization: a model and concept, Annals of the Association of American Geographers, Vol. 59, n 2 , p. 348-364.

J W., Wang Y., Zhuang D., Song D., Shen X., Wang W., Lı G., 2014, Spatial and temporal distribution of expressway and its relationships to land cover and population: A case study of Beijing, China, Transportation Research Part Transportation Environment, Vol. 32, p. 86-96.

KANSKY K. J., 1963, Structure of transportation networks, Chicago: University of Chicago.

Kasraian D., Maat K., Stead D., Van Wee B., 2016, Long-term impacts of transport infrastructure networks on landuse change: an international review of empirical studies, Transport Reviews, Vol 36, Issue 6, p. 1-21.

LePetit B., 1986, L'impensable réseau. Les routes françaises avant les chemins de fer, Cahiers du Groupe Réseaux, Vol. 2, n 5, p. 11-29. DOI : 10.3406/flux.1986.1084

Lévy J., Lussault M., 2003, Dictionnaire de la géographie et de l'espace des sociétés, Paris : Belin.

LhommE S., 2015, Analyse spatiale de la structure des réseaux techniques dans un contexte de risques, Cybergeo European Journal of Geography. DOI : 10.4000/cybergeo.26763

Martí-Henneberg J., 2013, Introduction to the Special Issue on the Common Ground of History and Geography, Historical Methods: A Journal of Quantitative and Interdisciplinarity History, Vol. 46, n 3, p. 113-116. DOI: 10.1080/01615440.2013.803395

Martí-HenneberG J., 2017, The influence of the railway network on territorial integration in Europe (1870-1950), Journal of Transport Geography, Vol. 62, p. 160-171. DOI: 10.1016/j. jtrangeo.2017.05.015

Mimeur C., 2016, Les traces de la vitesse entre réseau et territoire. Approche géohistorique de la croissance du réseau ferroviaire français, Thèse de géographie, Dijon : Bourgogne Franche-Comté.
Mimeur C., Queyroi F., Banos A., Thévenin T., 2018, Revisiting the structuring effect of transportation infrastructure: an empirical approach with the French Railway Network from 1860 to 1910, Historical Methods: A Journal of Quantitative and Interdisciplinarity History, Vol. 51, n 2, p. 65-81. DOI: 10.1080/01615440.2017.1393358

Morillas-Torné M., 2012, Creation of a Geo-Spatial Database to Analyse Railways in Europe (1830-2010). A Historical GIS Approach, Jounal of Geographic Information System, Vol. 04, n 02, p. 176-187. DOI: 10.4236/jgis.2012.42023

OfFNER J.-M., 1993, Les « effets structurants» du transport: mythe politique, mystification scientifique, Espace Géographique, Vol. 22, n³ 3, p. 233-242.

OfFNER J.-M., 2014, Les « effets structurants » du transport : vingt ans après, L'Espace Géographique, Vol. 43, n 1, p. 51-67.

Oluivro J., 2000, L'homme à toutes vitesses: de la lenteur homogène à la rapidité différenciée, Rennes : Presses universitaires de Rennes.

Pumain D., 1982, Chemin de fer et croissance urbaine en France au XIX siècle, Annales de Géographie, Vol. 91, n 507, p. 529-550. DOI : 10.3406/geo.1982.20140

Pumain D., 1997, Pour une théorie évolutive des villes, Espace Géographique, Vol. 26, n² 2, p. 119-134. DOI : 10.3406/ spgeo.1997.1063

PumAin D., 2014, Les effets structurants ou les raccourcis de l'explication géographique, L'Espace Géographique, Vol. 43, n 1, p. 51-67.

RibeiLl G., 1985, Aspects du développement du réseau ferré français sur la longue durée. L'approche historique, Cahiers du Groupe Réseaux, Vol. 1, n 1, p. 10-25. DOl :10.3406/ flux.1985.1595

Ribeill G., 1986, Au temps de la révolution ferroviaire. L'utopique réseau, Cahiers du Groupe Réseaux, Vol. 2, n 5, p. 48-59. DOI : 10.3406/flux.1986.1086

Riвoud J., 1981, La Ville heureuse : Doctrine et expériences de création urbaine, Paris : Moniteur.

Schwartz R., Gregory I. N., Marti-Henneberg J., 2011, History and GIS: Railways, population change, and agricultural development in late nineteenth-century Wales, in: Dear M., Ketchum J., Luria S., Richardson D. (eds.), GeoHumanities: Art, History, Text at the Edge of Place, New York: Routledge, p. 251-266.

Studeny C., 1995, L'invention de la vitesse: France, $X V I I l^{e}-X X^{e}$ siècle, Paris : Gallimard.

Thévenin T., Mimeur C., Schwartz R., Sapet L., 2016, Measuring one century of railway accessibility and population change in France. A historical GIS approach, Journal of Transport Geography, Vol. 56, p. 62-76. DOI: 10.1016/j.jtrangeo.2016.08.017

To W. M., 2016, Centrality of an Urban Rail System, Urban Rail Transit, Vol. 1, n 4, p. 249-256. DOI : 10.1007/s40864016-0031-3

XIE F., Levinson D., 2007, Measuring the Structure of Road Networks, Geographical Anaysis, Vol. 39, n³ 3, p. 336-356. DOI: $10.1111 /$ j.1538-4632.2007.00707.x

XIE F., Levinson D., 2008, The weakest link: The decline of the surface transportation network, Transportation Research Part E Logistics and Transportation. Review, Vol. 44, n 1, p. 100-113. 


\section{Résumé - Christophe Mimeur, Thomas Thévenin - Analyse diachronique de la croissance du réseau ferroviaire français entre 1860 et 1930 : entre ex- pansion connexionniste et sélection hiérarchique ?}

L'irruption du chemin de fer au cours du XIXe siècle en France a permis d'élargir l'horizon spatio-temporel en créant un choc d'accessibilité. Si l'infrastructure ferroviaire s'est largement diffusée dans sa phase de croissance dans l'ensemble du pays entre 1860 et 1930, la "révolution de la vitesse " permise par le train est loin d'être homogène. Cet article propose d'interroger ces deux dimensions du réseau ferroviaire français dans une perspective géohistorique et quantitative. II s'agit de montrer la force des phénomènes de hiérarchisation et de sélection dans le façonnement du réseau, tant d'un point de vue spatial que temporel. Pour cela, nous utilisons le formalisme des graphes grâce à la base de données FRANcE (French RAilway NEtwork), qui renferment des informations sur l'extension spatiale du réseau ainsi que sur les vitesses moyennes de chacun des tronçons. Nous utilisons la théorie des graphes pour identifier des principes universels de la science des réseaux et étudier leur inscription dans les territoires traversés. Ces résultats permettent de dresser une « fresque » du développement du chemin de fer : I'accès précurseur des places urbaines les plus dynamiques, l'identification d'axes structurants du réseau dont l'itinéraire peut parfois paraître surprenant, et la mise en place progressive de sous-réseaux. Ce travail participe à la construction d'un instrument de recherche permettant d'explorer les mutations socio-économiques à toutes les échelles, dont les temporalités peuvent être adaptées.

Mots-clés : Réseau, Géohistoire, Graphe, Modélisation, Ferroviaire

\begin{abstract}
Christophe Mimeur and Thomas Thévenin - Diachronic analysis of the growth of the French railway network between 1860 and 1930: connectionist expansion and hierarchical selection?
\end{abstract}

The emergence of the railways in France in the 19th century extended spatial and temporal horizons, creating a major disruption in accessibility. Although railway infrastructure spread widely in its growth phase throughout the country between 1860 and 1930, the 'speed revolution' made possible by the train was not homogeneous. This paper questions these two dimensions of the French railway network using a geohistorical and quantitative approach. Our aim is to show the impact of hierarchical selection processes in the shaping of the network, according to spatial and temporal perspectives. To this end, we use graph frameworks based on the French RAilway NEtwork (FRANCE) database which contains information on the spatial extension of the network and average speeds of network sections. We use graph theory to identify universal principles of network science and study their territorial inscription. These results retrace the development of the railway, showing that the most dynamic urban centres gained access first, and identifying the network's sometimes surprising structuring axes and the gradual establishment of sub-networks. This study contributes to the construction of a research instrument exploring socio-economic changes according to adaptive spatio-temporal scales.

Keywords: Network, Historical geography, Graphs, Modelling, Railways 